\title{
Predicting the frequency-dependent effective excess charge density: A new up-scaling approach for seismoelectric modeling
}

\author{
Damien Jougnot* and Santiago G. Solazzi ${ }^{\dagger}$ \\ *Sorbonne Université, CNRS, EPHE, UMR 7619 METIS, F-75005 Paris, France \\ ${ }^{\dagger}$ Institute of Earth Sciences, University of Lausanne, Lausanne, Switzerland.
}

(November 20, 2020)

GEO-2020-0524

Running head: Frequency-dependent effective excess charge

\begin{abstract}
The seismoelectric method is based on the capacity of seismic waves to generate measurable modifications of the electrical field in porous media. Even though it combines the advantages of both seismic and geoelectrical methods, it remains largely underused in hydrogeophysics. Its signal results from an electrokinetic coupling that can be modeled using either the coupling coefficient or the effective excess charge density. The traditional approach is based on the frequency-dependent coupling coefficient, which relates the pressure drop with the change in the electrical potential. A more recent approach consists of describing the excess charge that is effectively dragged by water flowing within the pores. We present a new model for the frequency dependent effective excess charge density. For this, we make use of a mechanistic up-scaling of the electrokinetic coupling in a capillary. This novel approach introduce inertial effects arising within the pore space in the flux-averaging procedure to explain the frequency dependence of the effective excess charge density. The presented
\end{abstract}


model is successfully compared to previous models and published data. This new frequencydependent up-scaling approach has the potential of fundamentally improving our current understanding of the seismoelectrical signal in more complex environments, such as partially saturated and fractured media. 


\section{INTRODUCTION}

Hydrogeophysical methods are quickly developing and can now be considered as a state of the art tools for critical zone studies (e.g., Binley et al., 2015). In the hydrogeophysicist tool box one can consider the geoeletrical methods, from DC (Direct Current) to higher frequencies electrical and electromagnetic methods (e.g., Revil et al., 2012; Kemna et al., 2012), and the seismic methods (e.g., Clair et al., 2015; Pasquet et al., 2016; Blazevic et al., 2020). However, only few works have been conducted on the use of the seismoelectrical method in the context of an hydrogeophysical study (e.g., Revil et al., 2015; Jouniaux and Zyserman, 2016). Among other works, one can cite the study of vadose zone hydrology (Dupuis et al., 2007; Strahser et al., 2011), glaciers (Kulessa et al., 2006), or borehole-based characterization of sediments (Dupuis and Butler, 2006).

Seismoelectrical signals arise by electrokinetic coupling from the propagation of a seismic wave in a charged porous medium. Constitutive minerals of geological media generally exhibit electrostatic charges at their surfaces, and, when in contact with a pore water electrolyte, an electrical double layer (EDL) develops at these interfaces. The EDL consists in an excess of electric charges in the pore water solution to compensate from the mineral surface charges. When a seismic wave propagates, it generates a relative displacement of the pore fluid with respect to the solid pore walls, moving the charges in solution with respect to the charges at the surface. This creates an electrical current and a resulting electrical field that can be measured remotely at the Earth's surface or within a geological medium and that, most importantly, contains information of interest for hydrogeophysical studies.

The study of physical processes underlying the seismoelectrical signal generation can be tracked back to the late 1930's (e.g., Thomson, 1939; Frenkel, 1944) but it remains an active 
research subject (e.g., Pride and Garambois, 2005; Revil et al., 2015; Jouniaux and Zyserman, 2016). The most traditional approach to model the seismoelectric conversion is the use of the electrokinetic coupling coefficient, that is, a frequency-dependent parameter that relates a difference in fluid pressure to a difference in electrical potential. The two most used frameworks to describe the evolution of the coupling coefficient as a function of frequency have been proposed by Packard (1953) and Pride (1994). These approaches have been able to reproduce experimental measurements of frequency-dependent streaming potential (e.g., Jouniaux and Bordes, 2012; Tardif et al., 2011) and seismoelectric measurements (e.g., Zhu and Toksöz, 2013).

More recently, following theoretical developments on streaming potentials (e.g., Kormiltsev et al., 1998; Revil and Leroy, 2004), a new modeling approach for seismoelectric was proposed by Revil and Jardani (2010). It consists in using the excess charge from the EDL as an electrokinetic coupling variable, that is, the excess of charges located in the diffuse layer which are effectively dragged by the relative displacement between the fluid and the solid part of the porous medium. This effective excess charge approach allows to directly relate the electrical current generated through electrokinetic coupling to the fluid movement itself (e.g., Jougnot et al., 2012; Revil and Jardani, 2013; Guarracino and Jougnot, 2018; Soldi et al., 2019). This approach has been used to model the seismoelectric conversion in reservoir rocks under saturated (e.g., Mahardika et al., 2012; Revil et al., 2015) and partially saturated conditions (e.g., Revil and Mahardika, 2013; Jardani and Revil, 2015) or the effect of wave-induced fluid flow (e.g., Jougnot et al., 2013; Monachesi et al., 2015; Rosas-Carbajal et al., 2020). Nevertheless, in most of these works, the effective excess charge density is considered to be independent from the frequency. In Revil and Mahardika (2013), the authors propose an empirical way to account for the dependence of this param- 
eter to the frequency, that is, they consider a relaxation time constant characterizing the transition between the viscous laminar flow regime to the inertial laminar flow regime of the Navier-Stokes equation. However, the microscopic characteristics relating the effective excess charge density with the frequency remain largely unexplored.

In this work, we present a novel approach that permits to up-scale the effective excess charge density from the pore scale including frequency dependent effects. For that, we make use of the flux-averaging framework proposed by Jougnot et al. (2012), in which the porous medium is conceptualized as a bundle of capillary tubes. First, we introduce the theoretical basis of the proposed model. Then, we analyze its senstivity, compare its results with the models of Packard (1953) and Revil and Mahardika (2013), and with experimental data from the literature.

\section{THEORETICAL DEVELOPMENT}

The proposed up-scaling procedure to obtain the effective excess charge density is summarized in Figure 1. First we describe the properties at the interface scale. Then we assume that, in the presence of a fluid pressure gradient, flow channels are generated within the pore space. These flow channels are conceptualized employing the capillary tube geometry. Models based on such approach have a long history (e.g., Kozeny, 1927) and, despite their conceptual simplicity, they have proven to be a highly effective tool for the realistic description of the hydraulic characteristics of porous media (for dynamic permeability, see Solazzi

et al., 2020). The proposed mechanistic description of oscillatory fluid flow processes, and the corresponding interaction with the EDL, result in a new model for the frequency dependent effective excess charge density at the pore scale. This parameter is then up-scaled at the REV scale following an assumption similar to the classical model of Packard (1953). 


\title{
Pore scale characterization
}

\author{
Oscillatory fluid motion in a capillary
}

Let us consider a capillary with a radius $R(\mathrm{~m})$ and a length $l(\mathrm{~m})$. Let $r(\mathrm{~m})$ be the distance from the pore wall $(r=0 \mathrm{~m})$ to the center of the capillary $(r=R)$. We shall also consider that the solid matrix is rigid and that the pore space is saturated with water, which is modeled as an incompressible Newtonian fluid with density $\rho_{w}\left(\mathrm{~kg} \mathrm{~m}^{-3}\right)$ and viscosity $\eta_{w}$ (Pa s). This capillary is submitted to a infinitesimal time-harmonic pressure gradient

$$
\Delta P(t)=\Delta P^{*} e^{-i \omega t}
$$

where $t(\mathrm{~s})$ is the time, $\Delta P^{*}(\mathrm{~Pa})$ is the pressure difference amplitude between the ends of the capillary, and $\omega$ is the angular frequency, which responds to $\omega=2 \pi f$, with $f(\mathrm{~Hz})$ the frequency, and $i$ is the complex unit. Parameters with ${ }^{*}$ denote amplitude variations of harmonic variables and, thus, following previous works in the matter, the harmonic term $e^{-i \omega t}$ is hereby dropped for ease notation (e.g., Johnson et al., 1987; Reppert et al., 2001). Note that the fluid can indeed be regarded as incompressible at the pore scale provided that the prevailing acoustic wavelengths in the fluid are much larger than the typical pore size (e.g., Johnson et al., 1987).

The water velocity as a function of the distance from the pore wall $r$ and angular frequency $\omega, v^{*}\left(\mathrm{~m} \mathrm{~s}^{-1}\right)$, can be obtained solving the Navier-Stokes equation, under the above described conditions, and yields

$$
v^{*}(r, \omega)=-\frac{1}{\eta_{w} \kappa^{2}}\left(\frac{J_{0}(\kappa(R-r))}{J_{0}(\kappa R)}-1\right) \frac{\Delta P^{*}}{l}
$$

where $\kappa^{2}=i \omega \frac{\rho_{w}}{\eta_{w}}$, and where $J_{\chi}$ are Bessel functions of the first kind of order $\chi$. The low-frequency limit of equation 2 (i.e., the quasi-static limit: $\omega \rightarrow 0 \mathrm{~Hz}$ ) corresponds to the 
Poiseuille model for laminar flow rate, that is,

$$
v^{*}(r)_{\text {Poise }}=\frac{1}{4 \eta_{w}}\left(R^{2}-(R-r)^{2}\right) \frac{\Delta P^{*}}{l},
$$

indicating that the velocity profile is parabolic. Note that equation 3 corresponds to the case studied by Jougnot et al. (2012). Integrating equation 2 over the cross-sectional area of the pore, we obtain the average water velocity $\bar{v}^{*}(\omega)$

$$
\bar{v}^{*}(\omega)=-\frac{1}{\eta_{w} \kappa^{2}}\left(\frac{2}{\kappa R} \frac{J_{1}(\kappa R)}{J_{0}(\kappa R)}-1\right) \frac{\Delta P^{*}}{l} .
$$

On can note here that the average water velocity within the capillary is complex and frequency dependent.

Figure 2 shows the distribution of the complex pore water velocity $v^{*}(r, \omega)$ within the capillary. Note that both amplitude (Figure 2a) and phase (Figure 2b) are obtained from equation 2, considering $\Delta P^{*} / l=1 \mathrm{~Pa} \mathrm{~m}^{-1}$. The maximum amplitude of the velocity corresponds to the lowest frequency and at the pore centre. For such a frequency limit the response approaches that of the Poiseuille model (equation 3). A transition frequency exists, above which, amplitude decreases with frequency. This is the so-called transition frequency $f_{t}(\mathrm{~Hz})$, which is given by (e.g., Solazzi et al., 2020)

$$
f_{t} \simeq \frac{\eta_{w}}{\pi \rho_{w} R^{2}}
$$

In the case displayed in Figure 2, that is $R=10^{-4} \mathrm{~m}$, the transition frequency is approximately $16 \mathrm{~Hz}$.

Figure 3 shows the evolution of the complex average water velocity $\bar{v}^{*}(\omega)$ with frequency for different capillary radii (obtained from equation 4 ). Again, we consider that $\Delta P^{*} / l=1$ $\mathrm{Pa} \mathrm{m}{ }^{-1}$. These spectra show that the maximum velocities are obtained for large $R$ at low frequencies. The absolute value of the velocity is constant for $f \ll f_{t}$ and equal to that 
associated with Poiseuille-type flow. As expected, water velocity decreases with increasing frequency from $f_{t}$, which can be clearly identified by the break in the curve in Figure $3 \mathrm{a}$ (equation 5). Again, the $f_{t}$ values increase with decreasing $R$.

\section{Electrical double layer description}

Let us consider that the capillary is saturated by a binary symmetric electrolyte (e.g., $\mathrm{NaCl}$ ) with a ionic concentration $C_{i}^{w}\left(\mathrm{~mol} \mathrm{~m}^{-3}\right)$ and valence $z_{i}= \pm 1$, where $i$ is the considered ion. The capillary inner surface (pore walls) have surface charges (e.g., negative charges for silicate and aluminosilicate minerals under typical conditions). Let us call co-ions the ions with the same charge as the surface (e.g., $\mathrm{Cl}^{-}$) and counter-ions the one with the opposite charge (e.g., $\mathrm{Na}^{+}$). In order to insure the electro-neutrality of the system, these surface charges are balanced by an excess of counter-ions in the pore water that are distributed in the so-called EDL. Figure 4a shows a sketch of the distribution of the charges in the EDL in the so-called Stern and diffuse layers. The Stern layer is a compact layer of counter-ions with a very restricted thickness (i.e., negligible compare to the pore size of typical soils and permeable rocks). The diffuse layer contains counter-ions and co-ions that can move but with a net excess of charge (e.g., positive in the silicate case: i.e. $C_{N a}>C_{C l}$ ). We assume that the interface between the Stern layer and the diffuse layer corresponds to the shear plane: the plane that separates the stationary fluid and the moving fluid (e.g., Hunter, 1981; Revil et al., 2002). We call zeta potential, $\zeta(\mathrm{V})$, the electrical potential along this plane. For a given mineral, this potential depends mainly on the ionic strength, the temperature, and the pH (e.g., Revil et al., 1999; Jaafar et al., 2009).

The distribution of ions in the pore water depends on the distribution of the local 
electrical potential $\psi=f(r)(\mathrm{V})$. Using the assumptions of Debye-Huc̈kel and considering a thin double layer (i.e., the thickness of the double layer is small compared to the pore size), Pride (1994) expresses the local potential distribution as a function of the distance from the mineral surface:

$$
\psi(r)=\zeta e^{\left(-\frac{r}{l_{D}}\right)}
$$

where $\zeta(\mathrm{V})$ is the Zeta potential (i.e. the electrical potential at the shear plane), $r$ is the distance from the shear plane (i.e., the pore wall as the Stern layer thickness is neglected), and $l_{D}$ is the Debye length, which is given by

$$
l_{D}=\sqrt{\frac{\varepsilon_{w} k_{B} T}{2 C_{N a C l}^{w} e_{0}^{2} N_{A}}},
$$

where $\varepsilon_{w}=\varepsilon_{r} \varepsilon_{0}(\mathrm{~F} \mathrm{~m}-1)$ is the pore water permittivity with the permittivity of vacuum $\varepsilon_{0}=8.854 \times 10^{-12} \mathrm{~F} \mathrm{~m}^{-1}$ and the relative permittivity of water $\varepsilon_{r}=80.1$ at $T=20{ }^{\circ} \mathrm{C}$, $k_{B}=1.381 \times 10^{-23} \mathrm{~J} \mathrm{~K}^{-1}$ is the Boltzmann constant, $T(\mathrm{~K})$ is the absolute temperature, $N_{A}=6.022 \times 10^{23}$ is the Avogadro number, and $e_{0}=1.6 \times 10^{-19} \mathrm{C}$ is the elementary charge. The thickness of the diffuse layer can be approximated as four Debye lengths $4 l_{D}$ (Hunter, 1981). Equation 6 is only valid for small surface charges and neglects the effects of the charges of the opposite capillary wall (for the case of overlapping diffuse layers, see Leroy and Maineult, 2018), which is a valid assumption for most aquifers in environmental conditions, i.e., permeable media and typical water chemical compositions (see discussion in Jougnot et al., 2019). The excess charge localized in the diffuse layer can be calculated by means of (e.g., Guarracino and Jougnot, 2018):

$$
\bar{Q}_{v}(r)=N_{A} e_{0} C_{N a C l}^{w}\left[e^{\left(-\frac{e_{0} \psi(r)}{k_{B} T}\right)}-e^{\left(\frac{e_{0} \psi(r)}{k_{B} T}\right)}\right] .
$$

Figure $4 \mathrm{~b}$ shows the distribution of the excess charge in the diffuse layer as a function of the distance from the pore wall $r$. One can see that $\bar{Q}_{v}(r) \rightarrow 0$ when the distance from the 
pore wall increases $\left(r \geq 4 l_{D}\right)$.

Frequency-dependent effective excess charge density

We extend the flux-averaging approach proposed by Jougnot et al. (2012) for Poiseuille flow accounting for inertial effects. The frequency dependent effective excess charge density is obtained from the distribution of the pore water flux $v^{*}(r, \omega)$ (equation 2, Figure 4c) and the distribution of the excess charge $\bar{Q}_{v}(r)$ (equation 8, Figure 4b). For this, we integrate the flux over the total area of the capillary and recover a complex-valued flux-averaged excess charge $\hat{Q}_{v}^{R, *}(R, \omega)\left(\mathrm{C} \mathrm{m}^{-3}\right)$, that is,

$$
\hat{Q}_{v}^{R, *}(R, \omega)=\frac{\int_{r=0}^{r=R} \bar{Q}_{v}(r) v^{*}(r, \omega)(R-r) d r}{\int_{r=0}^{r=R} v^{*}(r, \omega)(R-r) d r},
$$

which is the effective excess charge carried by the water flux $v^{*}(r, \omega)$ in the capillary. Equation 9 is one of the central methodological results of this paper as, for the first time in the literature, we consider the effective excess charge as a frequency dependent parameter $\hat{Q}_{v}^{R, *}(R, \omega)$ obtained through flux-averaging up-scaling. It is crucial to distinguish the total excess charge density $\bar{Q}_{v}$, which corresponds to all the charges in the diffuse layer (Figure $4 \mathrm{~b}$ ), and the effective (or dynamic) excess charge density $\hat{Q}_{v}^{*}(\omega)$, which responds to the charges that are effectively dragged by the pore water velocity $v^{*}(r, \omega)$ (see discussions in Revil and Mahardika, 2013; Jougnot et al., 2020, 2019). 


\section{Upscaled behavior of the frequency dependent effective excess charge at the REV scale}

At the Representative Elementary Volume (REV) scale, the electrokinetic coupling is usually described by the electrokinetic coupling coefficient:

$$
C_{E K}^{*}(\omega)=\frac{\Delta V}{\Delta P}
$$

which links $\Delta V(\mathrm{~V})$, the measurable electrical potential difference, and $\Delta P(\mathrm{~Pa})$, the imposed pressure difference at the boundaries of a considered rock sample (e.g., Jouniaux and Bordes, 2012). This parameter is frequency dependent and it can be described as follows:

$$
C_{E K}^{*}(\omega)=C_{E K}^{0} C_{E K}^{r e l, *}(\omega)
$$

where we call $C_{E K}^{0}\left(\mathrm{~V} \mathrm{~Pa}^{-1}\right)$ the quasi-static electrokinetic coupling coefficient (i.e., for sufficiently low frequencies $\left.\omega \ll \omega_{t}\right)$ and $C_{E K}^{r e l, *}(\omega)$ the frequency-dependent relative electrokinetic coupling coefficient, which decreases from 1 to 0 when the frequency increases above the transition frequency (e.g., Jouniaux and Bordes, 2012). This transition occurs at $\omega \simeq \omega_{t}=2 \pi f_{t}$, therefore:

$$
C_{E K}^{r e l, *}\left(\omega \ll \omega_{t}\right)=1
$$

For frequencies much smaller than $\omega_{t}$, the quasi-static electrokinetic coupling coefficient at the REV scale can be related to the effective excess charge in the electrical double layer, that is (e.g., Revil and Leroy, 2004; Revil and Mahardika, 2013)

$$
C_{E K}^{0}\left(\omega \ll \omega_{t}\right)=-\frac{\hat{Q}_{v}^{0} k^{0}}{\eta_{w} \sigma^{0}},
$$

where $\hat{Q}_{v}^{0}\left(\mathrm{C} \mathrm{m}^{-} 3\right)$ is the quasi-static effective excess charge of the medium at the REV scale, $\eta_{w}(\mathrm{~Pa} \mathrm{~s})$ is the dynamic viscosity of the pore fluid, and $k^{0}\left(\mathrm{~m}^{2}\right)$ and $\sigma^{0}\left(\mathrm{~S} \mathrm{~m}^{-1}\right)$ 
are the permeability and the electrical conductivity of the porous medium, respectively. A widely used approach to describe the electrical conductivity of a porous medium is given by:

$$
\sigma^{0}=\frac{\sigma_{w}}{F}+\sigma_{s}
$$

where $\sigma_{w}\left(\mathrm{~S} \mathrm{~m}^{-1}\right)$ is the electrical conductivity of the pore water that strongly depends on the concentration of ions in the pore water (e.g., Sen and Goode, 1992), $\sigma_{s}$ is the surface conductivity, $F(-)$ is the formation factor given by $F=\phi^{-m}$, with $\phi(-)$ the medium porosity and $m(-)$ the so-called cementation exponent (Archie, 1942).

Guarracino and Jougnot (2018) proposes an analytical model to predict the quasistatic effective excess charge density present in equation 13 :

$$
\hat{Q}_{v}^{0}=N_{A} e_{0} C_{N a C l}^{w} l_{D}^{2}\left[-2 \frac{e_{0} \zeta}{k_{B} T}-\left(\frac{e_{0} \zeta}{3 k_{B} T}\right)^{3}\right] \frac{1}{\tau^{2}} \frac{\phi}{k^{0}},
$$

where $\tau$ is the hydraulic tortuosity in the medium. Particularly, the hydraulic tortuosity of the medium from the porosity and the electrical formation factor are related by (Winsauer et al., 1952; Jougnot et al., 2020):

$$
\tau=\sqrt{F \phi}
$$

The permeability of a porous medium is also a frequency dependent parameter (e.g., Johnson et al., 1987; Pride, 1994), often called dynamic permeability, and it can be expressed as

$$
k^{*}(\omega)=k^{0} k^{r e l, *}(\omega)
$$

where $k^{r e l, *}(\omega)$ is the dynamic permeability relative to the value of $k^{0}$, such as $k^{r e l} \in[0 ; 1]$. Considering the porous medium is a bundle of capillaries having the same radius, Figure 3 illustrate the frequency behaviour of the dynamic permeability. The analogy of the bundle of capillaries having a given size has been used in previous models (e.g., Packard, 1953; Reppert et al., 2001; Solazzi et al., 2020). 
Using the similar analogy of the bundle of capillary, where one capillary size dominates the REV, we can consider that the frequency-dependent effective excess charge at the REV scale is

$$
\hat{Q}_{v}^{R E V, *}(\omega)=\hat{Q}_{v}^{0} \hat{Q}_{v}^{r e l, *}(\omega),
$$

where $\hat{Q}_{v}^{0}$ can be obtained using equation 15 , while $\hat{Q}_{v}^{r e l, *}(\omega)$ can be obtained numerically by solving equation 9 for the capillary size $R$ considered as representative of the porous medium.

The electrical conductivity $\sigma^{*}(\omega)$ can also be considered as a frequency dependent rock property, however this point will not be discussed in the present paper. For more information, one can refer to a very large on the subject (e.g., Chelidze and Gueguen, 1999; Jougnot et al., 2010; Revil, 2013).

Considering these assumptions, we can express the frequency dependent electrokinetic coupling coefficient as

$$
C_{E K}^{*}(\omega)=-\frac{\hat{Q}_{v}^{R E V, *}(\omega) k^{*}(\omega)}{\eta_{w} \sigma^{*}(\omega)}
$$

or

$$
C_{E K}^{*}(\omega)=C_{E K}^{0} \frac{\hat{Q}_{v}^{r e l, *}(\omega) k^{r e l, *}(\omega)}{\sigma^{r e l, *}(\omega)},
$$

and the relative electrokinetic coupling coefficient can be expressed as

$$
C_{E K}^{r e l, *}(\omega)=\frac{\hat{Q}_{v}^{r e l, *}(\omega) k^{r e l, *}(\omega)}{\sigma^{r e l, *}(\omega)}
$$

where the "rel" superscript refer to value of the corresponding parameter relative to the associated low frequency value (equation 13). 


\section{SENSITIVITY TEST OF THE MODEL}

\section{Effect of the pore size and the frequency on $\hat{Q}_{v}^{R, *}$}

In the proposed model, the first step is to compute the frequency dependent effective excess charge density $\hat{Q}_{v}^{R, *}$. This can be done by modifying the code proposed in Jougnot et al. (2012) and solving equation 9 numerically for each frequency $f$ and capillary radius $R$ iteratively.

Figure 5 shows the results of the numerical simulations to compute $\hat{Q}_{v}^{R \text {,* }}$ as a function of the capillary radius for frequencies between 1 and $10^{6} \mathrm{~Hz}$. As expected from the theory, the amplitude of the pore water velocity increases as the capillary radius increases for all the considered frequencies (Figure 5a) until the radius reaches a critical size related to the transition frequency (see equation 7). Following an opposite behavior, the effective excess charge density decreases as the capillary radius decreases (Figure 5b) as predicted and explained in the literature (e.g., Jougnot et al., 2012; Guarracino and Jougnot, 2018). Around the transition frequency $f_{t}$, the trend of $\hat{Q}_{v}^{R, *}(R, \omega)$ changes as its slopes reduces its steepness.

Figure 6 shows the same simulation results but as a function of the frequency to better understand the spectral behaviour of $\hat{Q}_{v}^{R, *}(R, \omega)$. Figures $6 \mathrm{a}$ and $6 \mathrm{~b}$ illustrate very clearly the effect of the transition frequency on the pore water velocity and the effective excess charge density, respectively. While the pore water velocity drops for $f \ll f_{t}$, the effective excess charge density increases by orders of magnitude. This behavior was predicted and discussed in Revil and Mahardika (2013) but not quantified mechanistically at the pore scale. To the best of the authors knowledge, this is the first time that this parameter is explicitly calculated as a function of frequency. Note that numerical instabilities start to 
appear in the code above $10^{5} \mathrm{~Hz}$ for the larger capillaries.

\section{Evolution of the frequency dependent coupling coefficient}

The second step in the proposed model is to introduce the computed relative pore water

permeability $k^{r e l, *}(\omega)$ and the relative effective excess charge density $\hat{Q}_{v}^{r e l, *}(\omega)$ in equation 21 to obtain the frequency dependent relative coupling coefficient. It implies that the porous medium can be described by a bundle of capillaries with a single radius size. Note that this assumption is one of the limitations of the model proposed in this study.

Figure 7 shows the model prediction of the amplitude of $k^{r e l, *}(\omega)$ (Figure $\left.7 \mathrm{a}\right), \hat{Q}_{v}^{r e l, *}(\omega)$ (Figure 7b), and the resulting coupling coefficient $C_{E K}^{r e l, *}(\omega)$ (Figure 7c). Note that for this example, the frequency dependence of the electrical conductivity is neglected (i.e., $\left.\sigma^{r e l, *}(\omega)=1\right)$. The results are presented relatively to their quasi-static value (i.e., $0 \mathrm{~Hz}$ ). The dynamic permeability decreases and effective excess charge density increases at higher frequencies. The ratio between these two parameters as a function of frequency yields the coupling coefficient, decreasing with the frequency. Note that the inflection on the curve does not occur at exactly the transition frequency, this can be seen quite clearly in the shape of the frequency dependent coupling coefficient as it decreases with the frequency. This behaviour has been previously discussed in the literature (e.g., Reppert et al., 2001,their Figure 2). 


\section{RESULTS AND DISCUSSION}

\section{Comparison with existing models}

As presented in Jouniaux and Bordes (2012), many models already exist to describe the frequency-dependent coupling coefficient. The two most used models are Packard (1953) and Pride (1994). Pride (1994) derives a coupling framework based on first principles and an upscaling approach based on volume averaging. On the other hand, Packard (1953) proposes a model which is based on a porous medium conceptualized as a bundle of capillaries having a singular capillary size, which is similar to the assumptions done in the model described in the previous section. The Packard (1953) model is expressed as follows:

$$
C_{E K}^{*}(\omega)=\left(\frac{\varepsilon \zeta}{\eta_{w} \sigma_{w}}\right)\left(\frac{2 \kappa}{R} \frac{J_{1}(R \kappa)}{J_{0}(R \kappa)} e^{-i \omega t}\right)
$$

Revil and Mahardika (2013) propose an alternative model to describe the frequencydependent coupling coefficient under the thin double layer approximation:

$$
C_{E K}^{*}(\omega)=\frac{C_{E K}^{0}}{\sqrt{1-i \omega \tau_{k}}}
$$

where the frequency dependence is mainly controlled by a relaxation time:

$$
\tau_{k}=\frac{k^{0} \rho_{w} F}{\mu_{w}}
$$

This last parameter is calculated using the values of $k^{0}$ and $F$ corresponding to a bundle of capillaries with a single size $R$ and the arbitrarily fixed porosity of $\phi=0.5$. Due to the straightness of the capillaries, $m=1$ yields $F=\phi^{-m}=2$.

Figure 8 shows the comparison between the proposed model (dotted lines) and the prediction from both Packard (1953) (plain lines) and Revil and Mahardika (2013) (dashed lines). One can see that the proposed model, which based on a numerical upscaling of the 
effective excess charge, reproduces exactly the predictions of the complex coupling coefficient from Packard (1953) for both amplitude (Figure 8a), real part (Figure 8b), and imaginary part (Figure 8c), for different pore radii from $10^{-6}$ to $10^{-3} \mathrm{~m}$. This behaviour was expected since the model of Packard (1953) is based on the same hypothesis than the one propose in this work, that is the medium can be approximated by an equivalent pore size. On the other hand, even though the overall results are fairly similar to those predicted by the model of Revil and Mahardika (2013), one can see that such model and the proposed approach exhibit slightly different values in the transition shape: i.e., the imaginary part of the model predicted by Revil and Mahardika (2013) is less than 5\% smaller than the one predicted by the proposed model. Nevertheless, these comparisons clearly show that the new upscaling procedure proposed to determine the frequency dependent effective excess charge density is consistent with existing models from the literature.

\section{Comparison with published data}

In the following, the model predictions are then compared with experimental seismoelectric laboratory data by Zhu and Toksöz (2013). They measured the quasi-static and the frequency-dependent coupling coefficient of a sandstone for five different pore-water conductivities and a frequency range from 50 to $120 \mathrm{kHz}$.

Figure 9a shows the predicted quasi-static effective excess charge using equation 15 for different $\mathrm{NaCl}$ concentrations in the pore water, that is $C_{N a C l}^{w}=0.0017,0.0085,0.0171$,

0.0342 , and $0.0684 \mathrm{~mol} \mathrm{~L}{ }^{-1}$. The corresponding zeta potentials are obtained using the approach of Revil et al. (1999) with the empirical parameters proposed by Jaafar et al. 
(2009):

$$
\zeta\left(C_{N a C l}^{w}\right)=-6.43+20.85 \log _{10}\left(\mathrm{C}_{\mathrm{NaCl}}^{\mathrm{w}}\right)
$$

The quasi-static permeability $k^{0}=4.44 \times 10^{-13} \mathrm{~m}^{2}$ and porosity $\phi=0.23$ of the sandstone have been measured by the authors. The only unknown parameter is the hydraulic tortuosity, which was set at $\tau=3.5$ as it was considered to be an acceptable value by Zhu et al. (2016). Note that Revil and Mahardika (2013) consider a fixed effective excess charge density of $\hat{Q}_{v}^{0}=1.4 \mathrm{C} \mathrm{m}^{-3}$.

Figure $9 \mathrm{~b}$ shows the prediction of the proposed model for the quasi-static coupling coefficient $C_{E K}^{0}$ based on equation 13 and the predictions from $\hat{Q}_{v}^{0}$ (Figure 9a). Note that, following the work of Revil and Mahardika (2013), we consider that the electrical conductivity can be described using equation 14 with $\sigma_{s}=1.2 \times 10^{-3} \mathrm{~S} \mathrm{~m}^{-1}$ the surface conductivity and $F=18$. One can see that the model reproduces fairly well the behavior of the coupling coefficient.

Figure 10a presents the evolution of the dynamic effective excess charge density calculated in an equivalent capillary radius $R=6.31 \times 10^{-6} \mathrm{~m}$ for five pore water conductivities of $0.012,0.048,0.095,0.18$, and $0.32 \mathrm{~S} \mathrm{~m}^{-1}$. Then, Figure 10b presents the results from equation 19 where $k^{r e l, *}(\omega)$ and $\hat{Q}_{v}^{r e l, *}(\omega)$ come from our model while $C_{E K}^{0}$ comes from the laboratory measurements by Zhu and Toksöz (2013) (considering $\sigma^{r e l, *}(\omega)=1$ ). Without any further parameter adjustments, the proposed model predicts very well the experimental data. 


\section{Outlook}

This new upscaling procedure, adapted from Jougnot et al. (2012) by taking into account the frequency dependent inertial viscous effects in the pore water flow, offers a large flexibility to be applied to any kind of complex media. The most straightforward development is to apply this procedure to the prediction of the coupling coefficient in partially saturated media (e.g., Bordes et al., 2015) through the use of the modeling approach of Solazzi et al. (2020). To the best of the authors knowledge, the only existing model is the one proposed by Revil and Mahardika (2013). The second extension will be toward an improved description of the frequency dependence of the effective excess charge density in fractured media (e.g., Zhu and Toksöz, 2005), which has not yet been explored.

\section{CONCLUSIONS}

The present work proposes a new mechanistic model to predict the frequency dependence of the coupling coefficient for an oscillatory flow. For this, we mechanistically define a frequency-dependent effective excess charge by taking into account the inertial terms in the Navier-Stokes equation at the pore scale. This new upscaling procedure reproduces the behaviour of two existing models that were obtained from completely different approaches. Finally, the proposed model reproduces very well published data at different pore water salinities. These results pave the way to an improved description of the frequency dependence of the effective excess charge density in complex media such as partially saturated media or fractured media and the development of the seismoelectric method as a more standard tool for hydrogeophysics studies. 


\section{ACKNOWLEDGMENTS}

The authors thank the Editor in chief, Jeffrey Shragge, the special section editor, Adam R. Mangel, and the three reviewers for their very nice and constructive comments. DJ warmly thanks Tobias Müller and Niklas Linde for their help at an early stage of this work. 


\section{REFERENCES}

Archie, G., 1942, The electrical resistivity log as an aid in determining some reservoir characteristics: Transaction of the american institute of mining and metallurgical engineers, $146,54-61$.

Binley, A., S. S. Hubbard, J. A. Huisman, A. Revil, D. A. Robinson, K. Singha, and L. D. Slater, 2015, The emergence of hydrogeophysics for improved understanding of subsurface processes over multiple scales: Water resources research, 51, 3837-3866.

Blazevic, L. A., L. Bodet, S. Pasquet, N. Linde, D. Jougnot, and L. Longuevergne, 2020, Time-lapse seismic and electrical monitoring of the vadose zone during a controlled infiltration experiment at the ploemeur hydrological observatory, france: Water, 12, 1230.

Bordes, C., P. Sénéchal, J. Barrière, D. Brito, E. Normandin, and D. Jougnot, 2015, Impact of water saturation on seismoelectric transfer functions: a laboratory study of coseismic phenomenon: Geophysical Journal International, 200, 1317-1335.

Chelidze, T., and Y. Gueguen, 1999, Electrical spectroscopy of porous rocks: A review-i. theoretical models: Geophysical Journal International, 137, 1-15.

Clair, J. S., S. Moon, W. Holbrook, J. Perron, C. Riebe, S. Martel, B. Carr, C. Harman, K. Singha, and D. d. Richter, 2015, Geophysical imaging reveals topographic stress control of bedrock weathering: Science, 350, 534-538.

Dupuis, J., and K. Butler, 2006, Vertical seismoelectric profiling in a borehole penetrating glaciofluvial sediments: Geophysical Research Letters, 33, L16301.

Dupuis, J. C., K. E. Butler, and A. W. Kepic, 2007, Seismoelectric imaging of the vadose zone of a sand aquifer: Geophysics, 72, A81-A85.

Frenkel, J., 1944, On the theory of seismic and seismoelectric phenomena in a moist soil: Journal of Physics, III, 230-241. 
Guarracino, L., and D. Jougnot, 2018, A physically based analytical model to describe effective excess charge for streaming potential generation in water saturated porous media: Journal of Geophysical Research: Solid Earth, 123, 52-65.

Hunter, R., 1981, Zeta potential in colloid science: Principles and applications: Academic Press. Colloid Science Series.

Jaafar, M. Z., J. Vinogradov, and M. D. Jackson, 2009, Measurement of streaming potential coupling coefficient in sandstones saturated with high salinity $\mathrm{NaCl}$ brine: Geophysical Research Letters, 36. (L21306).

Jardani, A., and A. Revil, 2015, Seismoelectric couplings in a poroelastic material containing two immiscible fluid phases: Geophysical Journal International, 202, 850-870.

Johnson, D., K. Koplic, and R. Dashen, 1987, Theory of dynamic permeability and tortuosity in fluid-saturated porous media: Journal of Fluid Mechanics, 176, 379-402.

Jougnot, D., A. Ghorbani, A. Revil, P. Leroy, and P. Cosenza, 2010, Spectral induced polarization of partially saturated clay-rocks: A mechanistic approach: Geophysical Journal International, 180, 210-224.

Jougnot, D., N. Linde, A. Revil, and C. Doussan, 2012, Derivation of soil-specific streaming potential electrical parameters from hydrodynamic characteristics of partially saturated soils: Vadose Zone Journal, 11.

Jougnot, D., A. Mendieta, P. Leroy, and A. Maineult, 2019, Exploring the effect of the pore size distribution on the streaming potential generation in saturated porous media, insight from pore network simulations: Journal of Geophysical Research: Solid Earth, 124, 5315-5335.

Jougnot, D., D. Roubinet, L. Guarracino, and A. Maineult, 2020, Modeling streaming potential in porous and fractured media, description and benefits of the effective excess 
charge density approach, in Advances in Modeling and Interpretation in Near Surface Geophysics: Springer Geophysics Series.

Jougnot, D., J. G. Rubino, M. Rosas-Carbajal, N. Linde, and K. Holliger, 2013, Seismoelectric effects due to mesoscopic heterogeneities: Geophysical Research Letters, 40, 2033-2037.

Jouniaux, L., and C. Bordes, 2012, Frequency-dependent streaming potentials: a review: International Journal of Geophysics, 2012, 648781.

Jouniaux, L., and F. Zyserman, 2016, A review on electrokinetically induced seismoelectrics, electro-seismics, and seismo-magnetics for earth sciences: Solid Earth, 7, 249284.

Kemna, A., A. Binley, G. Cassiani, E. Niederleithinger, A. Revil, L. Slater, K. H. Williams, A. F. Orozco, F.-H. Haegel, A. Hoerdt, et al., 2012, An overview of the spectral induced polarization method for near-surface applications: Near Surface Geophysics, 10, 453-468.

Kormiltsev, V. V., A. N. Ratushnyak, and V. A. Shapiro, 1998, Three-dimensional modeling of electric and magnetic fields induced by the fluid flow movement in porous media: Physics of the earth and planetary interiors, 105, 109-118.

Kozeny, J., 1927, Über kapillare leitung des wassers im boden:(aufstieg, versickerung und anwendung auf die bewässerung): Hölder-Pichler-Tempsky.

Kulessa, B., T. Murray, and D. Rippin, 2006, Active seismoelectric exploration of glaciers: Geophysical Research Letters, 33, L07503.

Leroy, P., and A. Maineult, 2018, Exploring the electrical potential inside cylinders beyond the debye-hückel approximation: a computer code to solve the poisson-boltzmann equation for multivalent electrolytes: Geophysical Journal International, 214, 58-69.

Mahardika, H., A. Revil, and A. Jardani, 2012, Waveform joint inversion of seismograms 
and electrograms for moment tensor characterization of fracking events: Geophysics, $\mathbf{7 7}$, ID23-ID39.

Monachesi, L. B., J. G. Rubino, M. Rosas-Carbajal, D. Jougnot, N. Linde, B. Quintal, and K. Holliger, 2015, An analytical study of seismoelectric signals produced by 1-d mesoscopic heterogeneities: Geophysical Journal International, 201, 329-342.

Packard, R. G., 1953, Streaming potentials across glass capillaries for sinusoidal pressure: The Journal of Chemical Physics, 21, 303-307.

Pasquet, S., W. Holbrook, B. Carr, and K. Sims, 2016, Geophysical imaging of shallow degassing in a yellowstone hydrothermal system: Geophysical Research Letters, 43, 12027.

Pride, S., 1994, Governing equations for the coupled electromagnetics and accoustics of porous media: Physical Review B, 50, 15678-15696.

Pride, S., and S. Garambois, 2005, Electroseismic wave theory of Frenkel and more recent developments: Journal of Engineering Mechanics-ASCE, 131, 898-907.

Reppert, P., F. Morgan, D. Lesmes, and L. Jouniaux, 2001, Frequency-dependent streaming potentials: Journal of colloid and interface science, 234, 194-203.

Revil, A., 2013, Effective conductivity and permittivity of unsaturated porous materials in the frequency range $1 \mathrm{mhz}-1$ ghz: Water Resources Research, 49, 306-327.

Revil, A., D. Hermitte, E. Spangenberg, and J. Cochemé, 2002, Electrical properties of zeolitized volcaniclastic materials: Journal of geophysical research, 107, ECV 3-1-ECV 3-17.

Revil, A., and A. Jardani, 2010, Stochastic inversion of permeability and dispersivities from time lapse self-potential measurements: a controlled sandbox study: Geophysical Research Letters, 37, L11404. 
— , 2013, The self-potential method: Theory and applications in environmental geosciences: Cambridge University Press.

Revil, A., A. Jardani, P. Sava, and A. Haas, 2015, The seismoelectric method: Theory and application: John Wiley \& Sons, Ltd.

Revil, A., M. Karaoulis, T. Johnson, and A. Kemna, 2012, Review: Some low-frequency electrical methods for subsurface characterization and monitoring in hydrogeology: Hydrogeology Journal, 1-42.

Revil, A., and P. Leroy, 2004, Constitutive equations for ionic transport in porous shales: Journal of Geophysical Research, 109, B03208.

Revil, A., and H. Mahardika, 2013, Coupled hydromechanical and electromagnetic disturbances in unsaturated porous materials: Water Resources Research, 49, 744-766.

Revil, A., P. Pezard, and P. Glover, 1999, Streaming potential in porous media: 1. theory of the zeta potential: Journal of Geophysical Research: Solid Earth, 104, 20021-20031.

Rosas-Carbajal, M., D. Jougnot, J. G. Rubino, L. B. Monachesi, N. Linde, and K. Holliger, 2020, Seismoelectric signals produced by mesoscopic heterogeneities: spectroscopic analysis of fractured media, in Seismoelectric exploration: Theory, experiments and applications: Wiley.

Sen, P. N., and P. A. Goode, 1992, Influence of temperature on electrical conductivity on shaly sands: Geophysics, 57, 89-96.

Solazzi, S. G., J. G. Rubino, D. Jougnot, and K. Holliger, 2020, Dynamic permeability functions for partially saturated porous media: Geophysical Journal International, 221, $1182-1189$.

Soldi, M., D. Jougnot, and L. Guarracino, 2019, An analytical effective excess charge density model to predict the streaming potential generated by unsaturated flow: Geophysical 
Journal International, 216, 380-394.

Strahser, M., L. Jouniaux, P. Sailhac, P. Matthey, and M. Zillmer, 2011, Dependence of seismoelectric amplitudes on water content: Geophysical Journal International, 187, $1378-1392$.

Tardif, E., P. W. Glover, and J. Ruel, 2011, Frequency-dependent streaming potential of ottawa sand: Journal of Geophysical Research: Solid Earth, 116, B04206.

Thomson, R., 1939, A note on the seismic-electric phenomenon: Geophysics, 4, 102-105.

Winsauer, W. O., H. Shearin Jr, P. Masson, and M. Williams, 1952, Resistivity of brinesaturated sands in relation to pore geometry: AAPG bulletin, 36, 253-277.

Zhu, Z., and M. Toksöz, 2005, Seismoelectric and seismomagnetic measurements in fractured borehole models: GEOPHYSICS, 70, F45-F51.

Zhu, Z., M. Toksöz, and X. Zhan, 2016, Seismoelectric measurements in a porous quartzsand sample with anisotropic permeability: Geophysical Prospecting, 64, 700-713.

Zhu, Z., and M. N. Toksöz, 2013, Experimental measurements of the streaming potential and seismoelectric conversion in berea sandstone: Geophysical Prospecting, 61, 688-700. 


\section{LIST OF FIGURES}

1 Flow chart describing the procedure used in this work to to up-scale the frequency dependent effective excess charge.

2 (a) Absolute value and (b) phase of the pore water velocity $v^{*}(r, \omega)$ as functions of the distance from the pore wall. We consider different frequencies, which are denoted by different colors. We considered here $R=10^{-4} \mathrm{~m}$ and $\Delta P^{*} / l=1 \mathrm{~Pa} \mathrm{~m}^{-1}$ (equation 2). For reference we also illustrate the behaviour for Poiseuille flow (equation 3).

3 (a) Absolute value and (b) phase of the complex average pore water velocity as functions of frequency for pore radii varying between $10^{-8} \mathrm{~m}$ and $10^{-3} \mathrm{~m}$.

4 (a) Sketch of the electrical double layer. (b) Static excess charge density (for $C_{N a C l}^{w}=10^{-4} \mathrm{~mol} \mathrm{~L}^{-1}$ and $\zeta=-89.8 \mathrm{mV}$ ). (c) Amplitude of the pore velocity distribution as a function of the distance from the pore wall in a capillary $\left(R=1.26 \times 10^{-4} \mathrm{~m}\right)$. The vertical dashed layer across the (b) and (c) subplots corresponds to a distance of $r=4 l_{D}$.

5 Amplitude of (a) the water flux and (b) the effective excess charge density calculated with the model as a function of capillary radii for different frequencies. The dashed black line correspond a Poiseuille-type behavior of the corresponding variable, i.e., the case presented in Jougnot et al. (2012) for $f=0 \mathrm{~Hz}$.

6 Amplitude spectra of (a) the water flux and (b) the effective excess charge density calculated with the proposed model as functions of frequency for different capillary radii.

$7 \quad$ Predicted amplitude of (a) the relative dynamic permeability, (b) the relative dynamic effective excess charge density, and (c) the relative electrokinetic coupling coefficient calculated with the model as functions of frequency for different capillary radii (from $10^{-3}$ to $\left.10^{-6} \mathrm{~m}\right)$.

8 Predicted (a) amplitude, (b) real part, and (c) imaginary part of the relative cou- 
pling coefficient by two models for different radii (from $10^{-3}$ to $10^{-6} \mathrm{~m}$ ). The solid and dashed lines correspond to the model by Packard (1953) and Revil and Mahardika (2013), respectively, while the dots correspond to the proposed model.

9 (a) Quasi-static effective excess charge density and (b) coupling coefficient prediction of the proposed model for the sandstone sample used by Zhu and Toksöz (2013) and comparison with their experimental data (circles).

10 (a) Amplitude of the effective excess charge density as a function of the frequency from the proposed model for different pore water conductivities. Comparison between the amplitude measured coupling coefficient by Zhu and Toksöz (2013) (colored circles) and the model predictions (colored lines). 


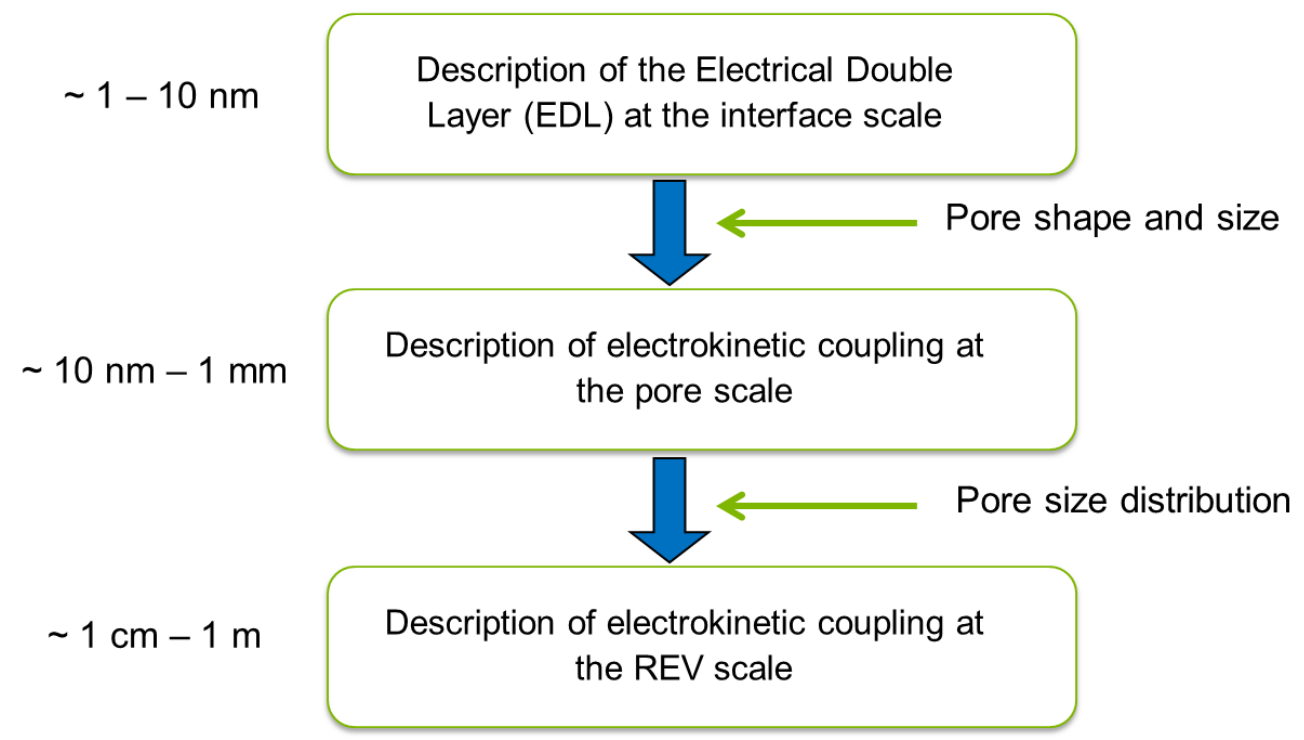

Figure 1: Flow chart describing the procedure used in this work to to up-scale the frequency dependent effective excess charge.

Jougnot \& Solazzi - GEO-2020-0524 

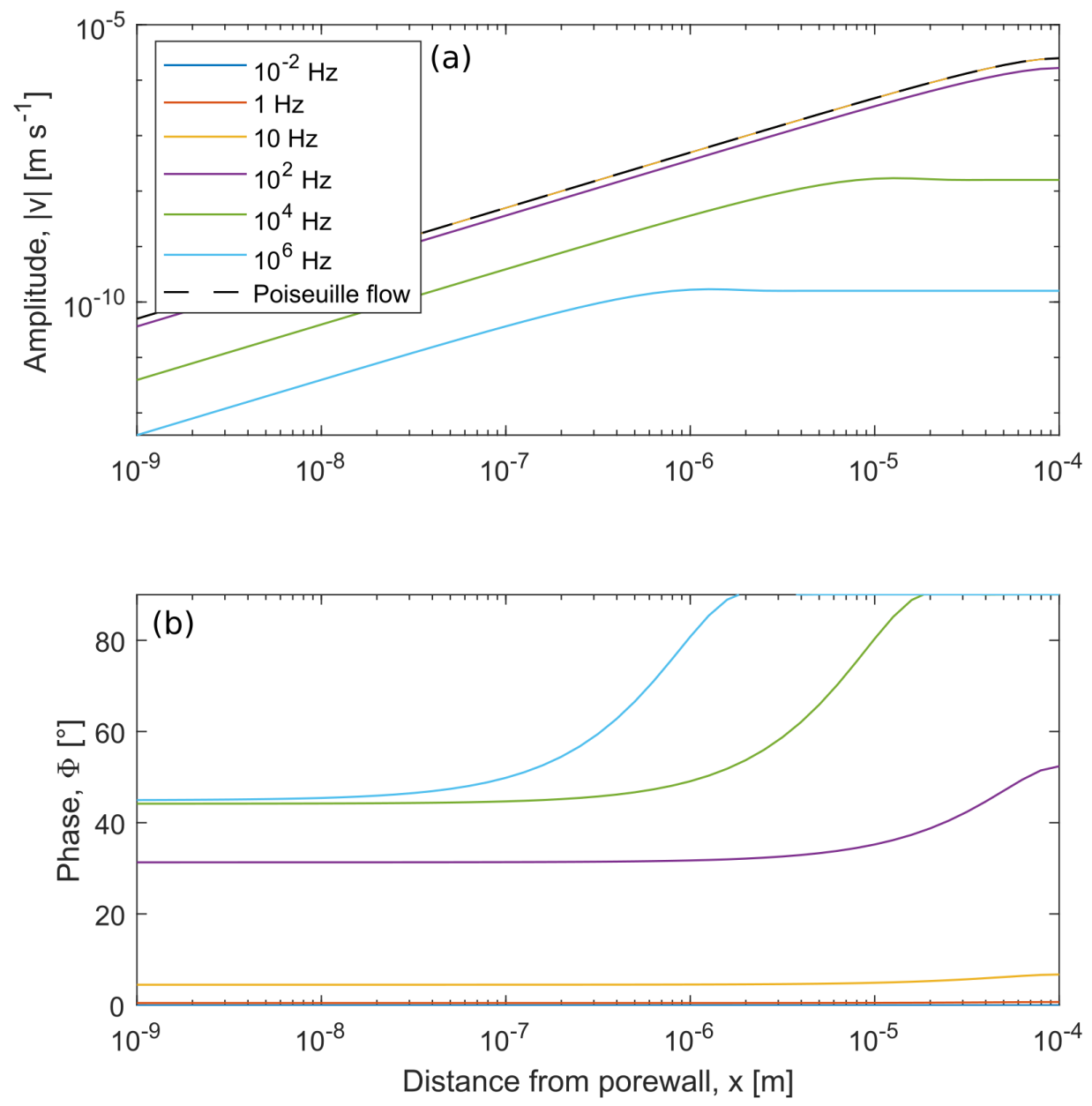

Figure 2: (a) Absolute value and (b) phase of the pore water velocity $v^{*}(r, \omega)$ as functions of the distance from the pore wall. We consider different frequencies, which are denoted by different colors. We considered here $R=10^{-4} \mathrm{~m}$ and $\Delta P^{*} / l=1 \mathrm{~Pa} \mathrm{~m}^{-1}$ (equation 2). For reference we also illustrate the behaviour for Poiseuille flow (equation 3).

\section{Jougnot \& Solazzi - GEO-2020-0524}



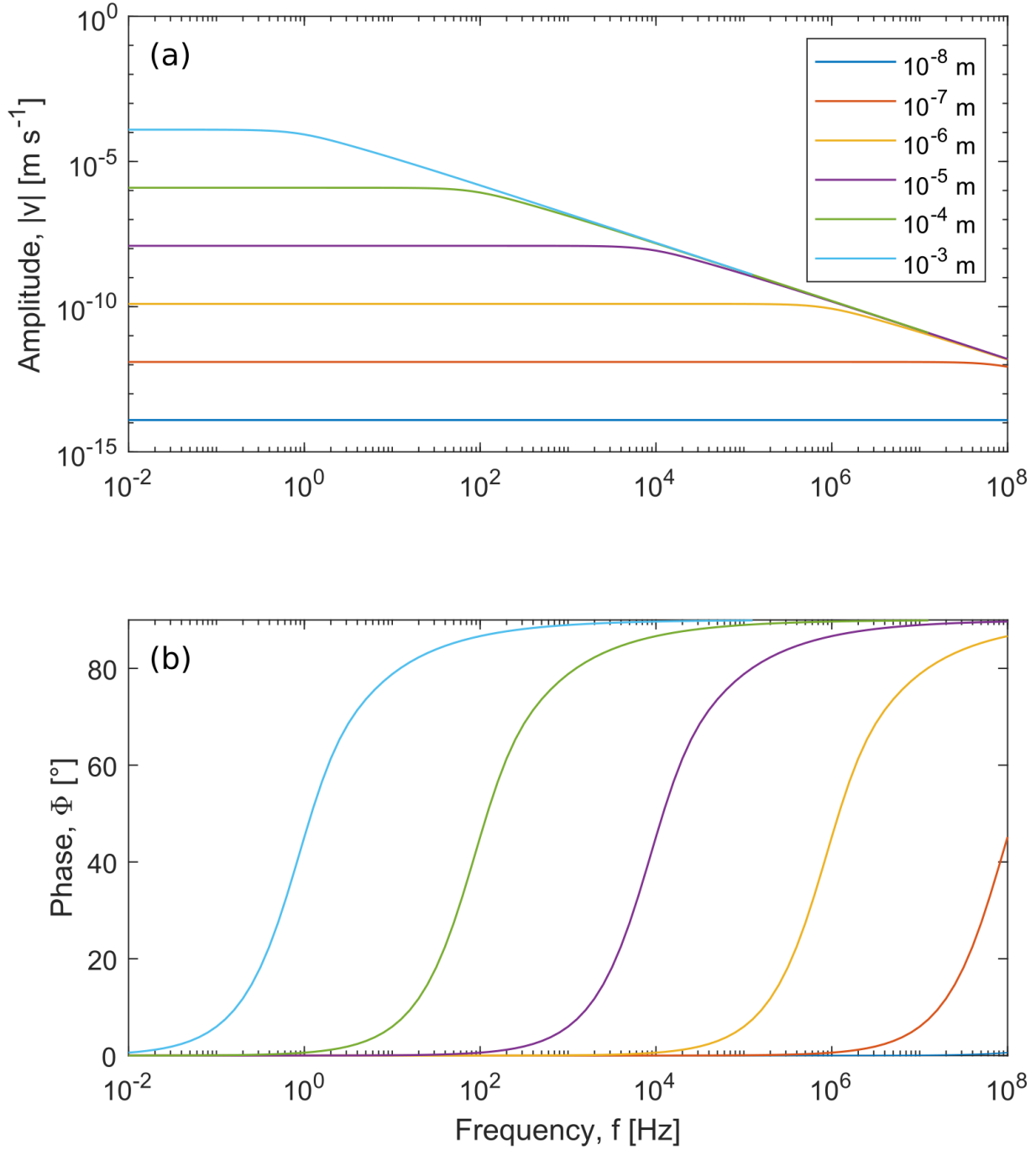

Figure 3: (a) Absolute value and (b) phase of the complex average pore water velocity as functions of frequency for pore radii varying between $10^{-8} \mathrm{~m}$ and $10^{-3} \mathrm{~m}$.

\section{Jougnot \& Solazzi - GEO-2020-0524}



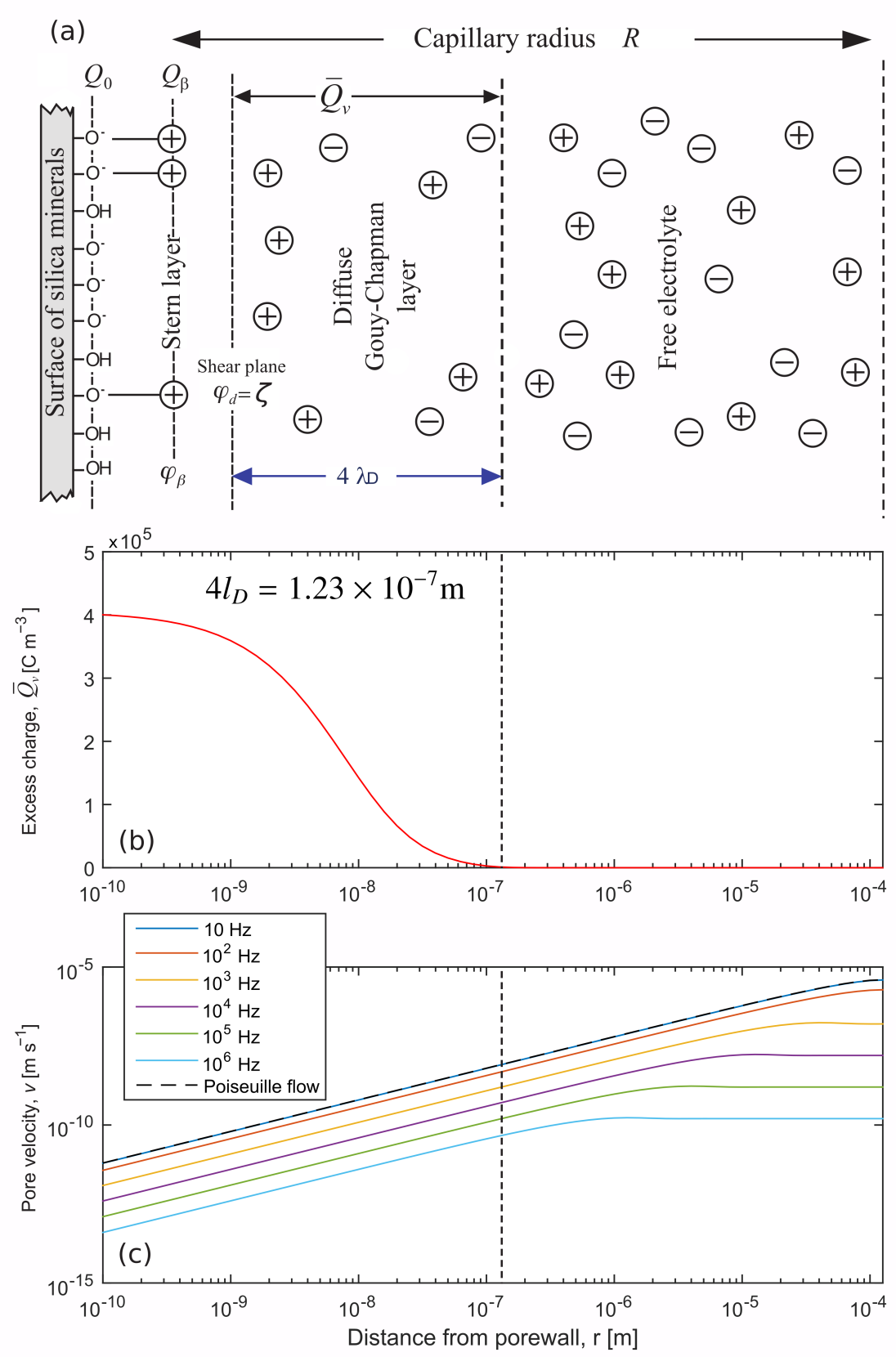

Figure 4: (a) Sketch of the electrical double layer. (b) Static excess charge density (for $C_{N a C l}^{w}=10^{-4} \mathrm{~mol} \mathrm{~L}^{-1}$ and $\zeta=-89.8 \mathrm{mV}$ ). (c) Amplitude of the pore velocity distribution as a function of the distance from the pore wall in a capillary $\left(R=1.26 \times 10^{-4} \mathrm{~m}\right)$. The vertical dashed layer across the (b) and (c) subplots corresponds to a distance of $r=4 l_{D}$. 

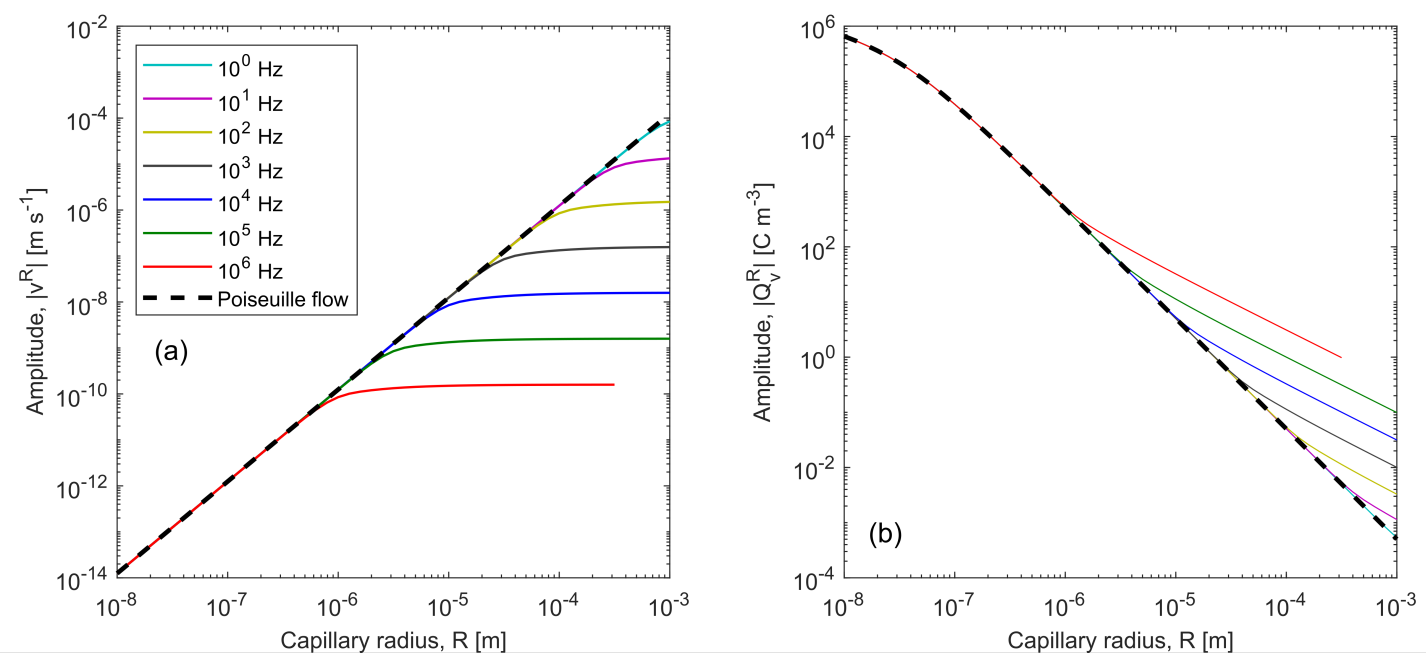

Figure 5: Amplitude of (a) the water flux and (b) the effective excess charge density calculated with the model as a function of capillary radii for different frequencies. The dashed black line correspond a Poiseuille-type behavior of the corresponding variable, i.e., the case presented in Jougnot et al. (2012) for $f=0 \mathrm{~Hz}$.

\section{Jougnot \& Solazzi - GEO-2020-0524}



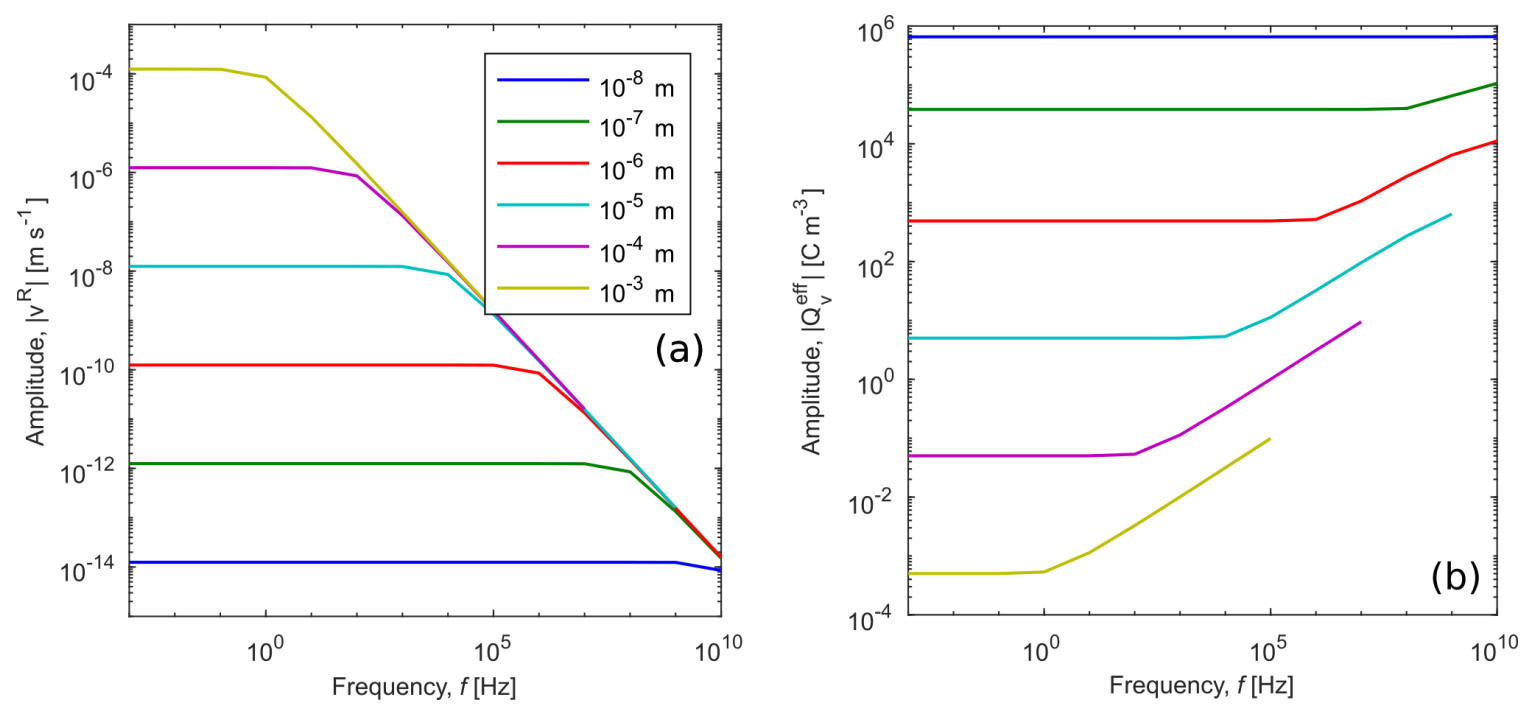

Figure 6: Amplitude spectra of (a) the water flux and (b) the effective excess charge density calculated with the proposed model as functions of frequency for different capillary radii.

\section{Jougnot \& Solazzi - GEO-2020-0524}



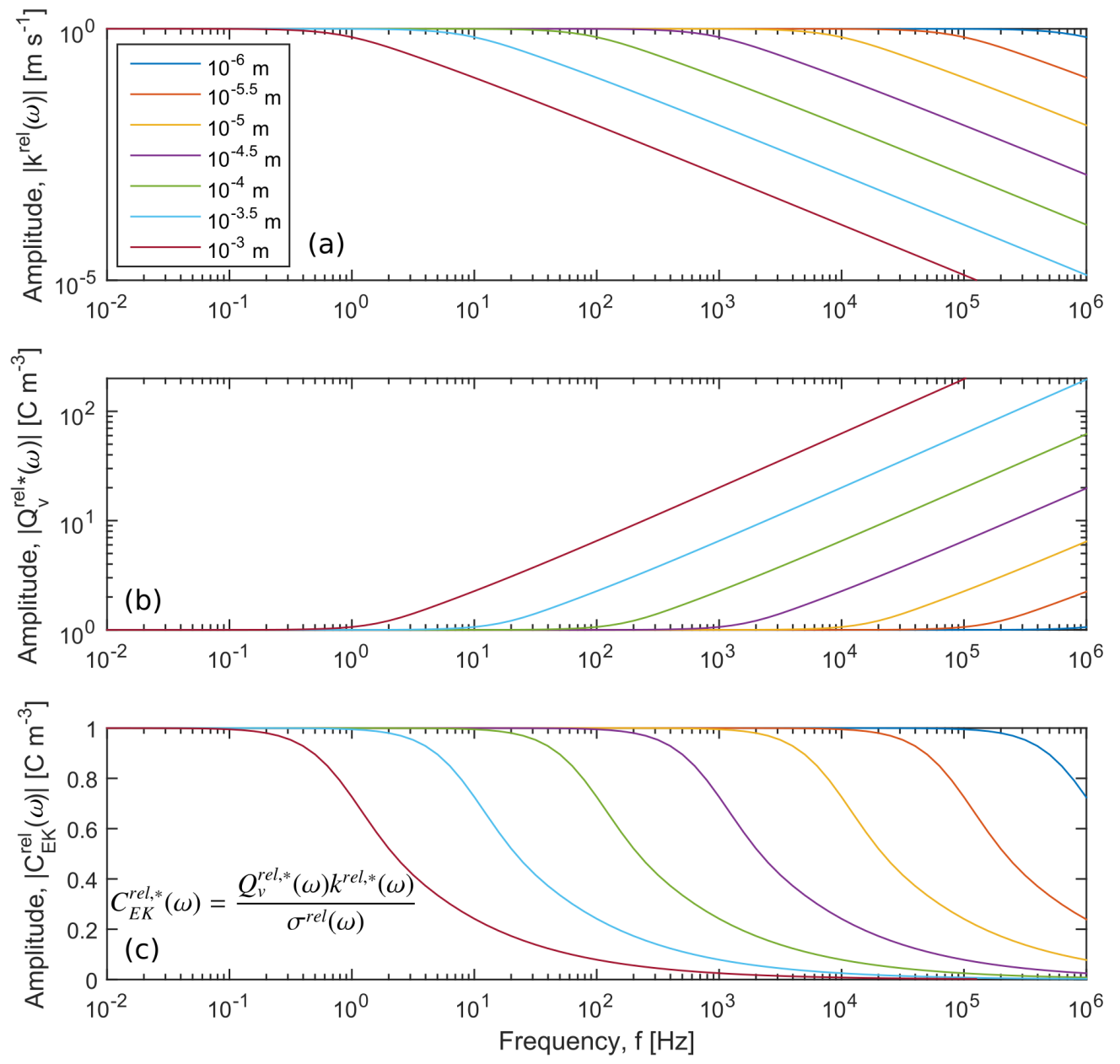

Figure 7: Predicted amplitude of (a) the relative dynamic permeability, (b) the relative dynamic effective excess charge density, and (c) the relative electrokinetic coupling coefficient calculated with the model as functions of frequency for different capillary radii (from $10^{-3}$ to $\left.10^{-6} \mathrm{~m}\right)$.

Jougnot \& Solazzi - GEO-2020-0524 

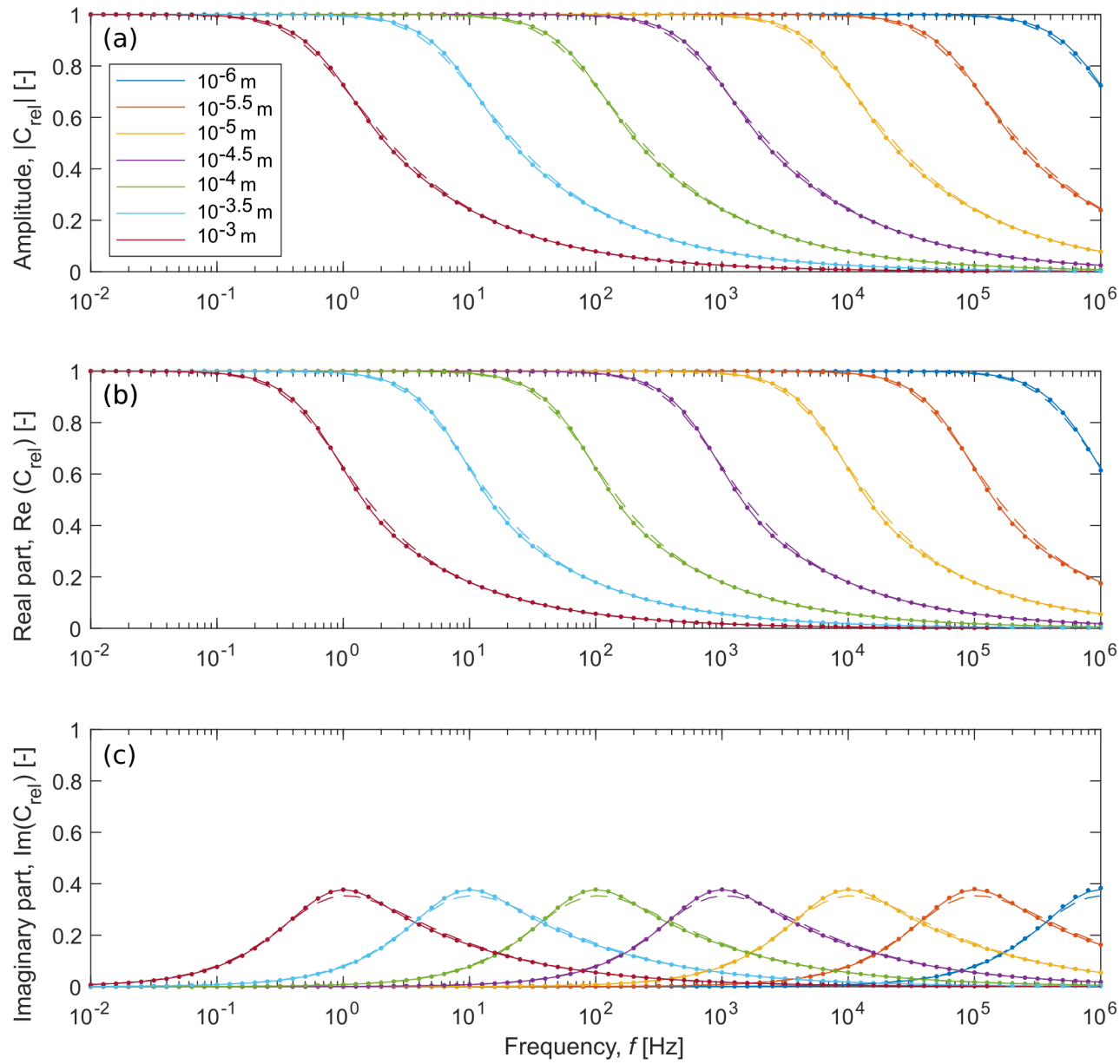

Figure 8: Predicted (a) amplitude, (b) real part, and (c) imaginary part of the relative coupling coefficient by two models for different radii (from $10^{-3}$ to $10^{-6} \mathrm{~m}$ ). The solid and dashed lines correspond to the model by Packard (1953) and Revil and Mahardika (2013), respectively, while the dots correspond to the proposed model.

Jougnot \& Solazzi - GEO-2020-0524 

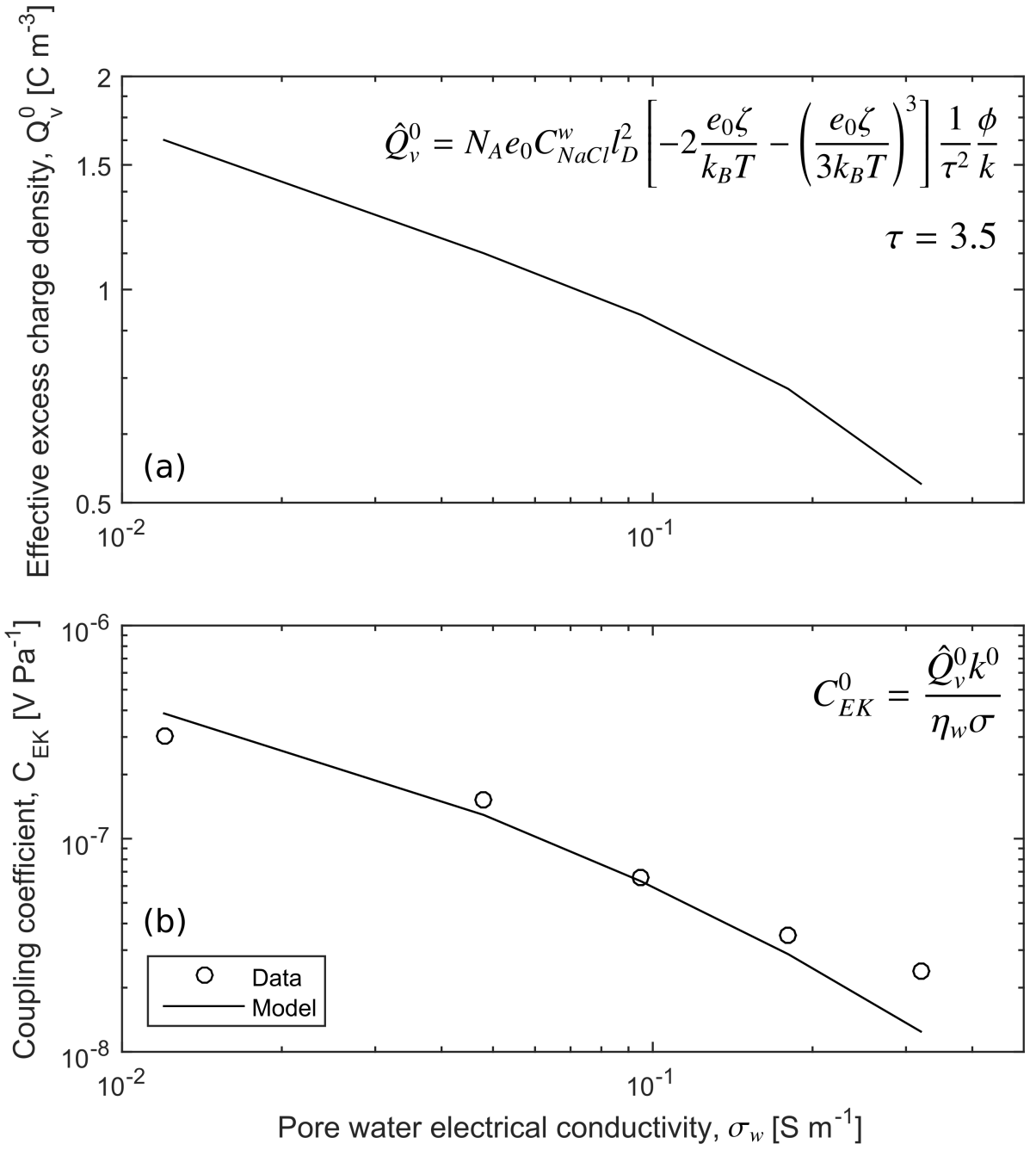

Figure 9: (a) Quasi-static effective excess charge density and (b) coupling coefficient prediction of the proposed model for the sandstone sample used by Zhu and Toksöz (2013) and comparison with their experimental data (circles).

Jougnot \& Solazzi - GEO-2020-0524 

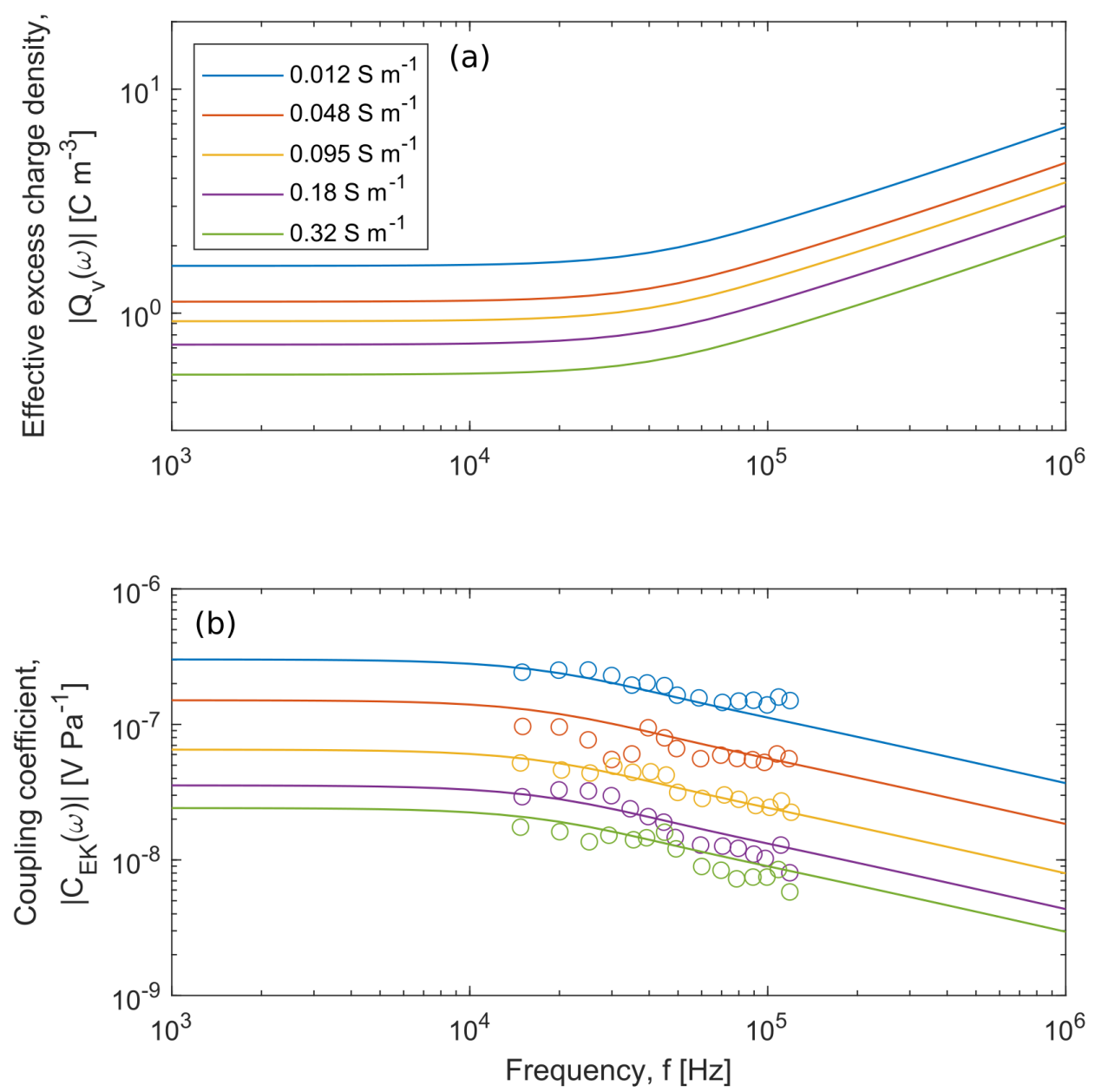

Figure 10: (a) Amplitude of the effective excess charge density as a function of the frequency from the proposed model for different pore water conductivities. Comparison between the amplitude measured coupling coefficient by Zhu and Toksöz (2013) (colored circles) and the model predictions (colored lines).

Jougnot \& Solazzi - GEO-2020-0524 